\title{
Continuous-Time Adaptive Delay System
}

\author{
Shih-Chii Liu and Carver Mead \\ California Institute of Technology \\ Computational and Neural Systems Program \\ Pasadena, California 91125, U.S.A.
}

\begin{abstract}
We have developed an adaptive delay system that adjusts the delay of a delay element so that it matches the temporal disparity between the onset of two input signals. The delay is controlled either by an external bias voltage, or by an intrinsic signal derived from an adaptive block. The operation of the adaptive delay system is similar to that of a charge-pump phase-lock loop, with an extended lock-in range of more than 5 decades. Standard CMOS transistors are used in their subthreshold region. Experimental results from circuits fabricated in $2 \mu \mathrm{m}$ CMOS technology are in agreement with the analysis.
\end{abstract}

\section{Introduction}

Conventional charge-pump phase-locked loop systems compare the output of a voltage controlled oscillator (VCO) to an incoming periodic input signal[1]. A phase comparator circuit compares the relative timing of the VCO output and the input signal and generates a lead or lag signal with a duration corresponding to the time difference between the onset of the VCO and that of the input. The lead or lag signal (error signal) is used to correct the voltage input to the VCO so that the correct VCO frequency is generated. The voltage input is stored on a capacitor and the capacitor is charged or discharged with a fixed current source during the duration of the error signal. The VCO frequency can show considerable overshoot before it settles to the correct frequency.

The adaptive delay system described here incorporates a versatile delay element that has continuous-time adaptation. The delay through a delay element is controlled by a bias voltage. The system computes the temporal disparity between the onset of two input signals and adapts the bias voltage so that the delay through the delay element matches the temporal disparity of the signals. The progression of the bias voltage to the final steady-state value occurs monotonically, and the gain of the system can be adjusted to control the time constant of this adaptation. A variable current source controlled by the bias voltage is used to charge or discharge the capacitor on which this voltage is stored. Their new adaptation dynamics allow the lock-in range of the adaptive system to occur over 5 decades in delay. This adaptive system is similar to the adaptation characteristics in biological systems that must handle input signals with a large dynamic range in time or other dimensions.

The circuit was fabricated in $2 \mu m$ CMOS technology: experimental results from the chip are presented. The adaptive delay capability can be incorporated in any system that includes a delay element.

\section{Architectural Model}

A block diagram of the adaptive delay system is shown in Figure 1. The relative timing of the signals in this system is shown in Figure 2. The signals shown are all discrete-level, continuous-time pulses except for the bias voltage, $V_{D}$. The signal pulse 1 is the input signal that arrives first while pulse 2 is the input signal that arrives next. The interval $t_{i}$ is the input temporal disparity between both input signals. The signal dpulse 1 is a delayed version of pulsel where the delay of the variable delay element, $t_{d}$, is controlled by $V_{D}$. Circuits using the signals, pulse1, pulse 2 and $d p u l s e 1$, are leading edge-triggered. The lead/lag discriminator in Figure 1 compares the arrival of $d p u l s e 1$ with that of pulse2 and outputs an error signal, $V_{\text {lead }}$ or $V_{\text {lag }}$ if the signals do not arrive in synchrony. The feedback system uses $V_{\text {lead }}$ and $V_{\text {lag }}$, to correct $V_{D}$ in the charge pump block in Figure 1. This change in $V_{D}$ is reflected as a change of $t_{d}$ in the variable delay element so that $t_{d}$ adapts to $t_{i}$.

\section{Lead/Lag Discriminator}

The lead/lag discriminator circuit shown in Figure 3 computes the time difference $\Delta t$ between the delay through the delay element, $t_{d}$ and the input temporal disparity, $t_{i}$. $V_{l a g}$ becomes active for the duration $\Delta t$ when $t_{i}<t_{d}$ and $\overline{V_{\text {lead }}}$ becomes active for the duration $\Delta t$ when $t_{i}>t_{d}$. The circuit on the right of Figure 3 


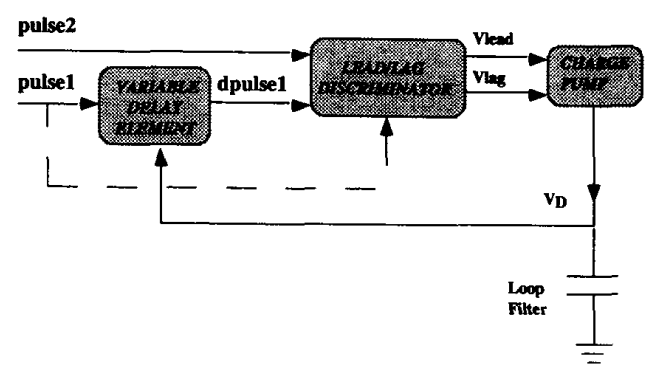

Figure 1: Block diagram of adaptive delay system. All signals are pulses (continuous-time, discrete-level) except for the bias voltage, $V_{D}$.

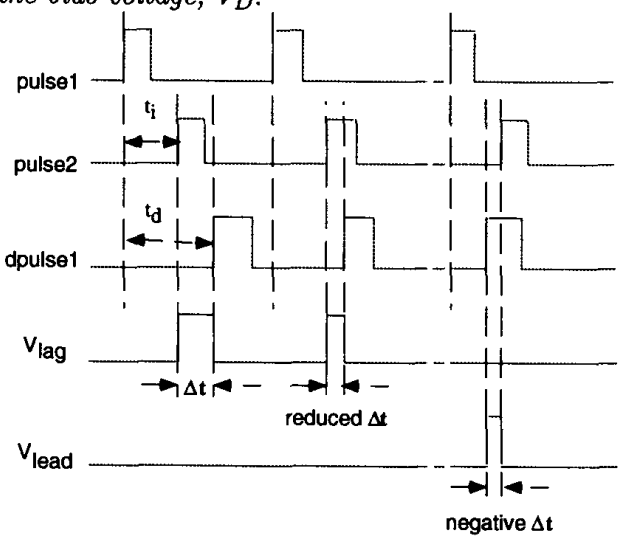

Figure 2: Relative timing of the signals shown in the block diagram in Figure 1. The input signals pulse1, pulse 2 and dpulse 1 are edge-triggered. The system adapts $t_{d}$ to match $t_{i}$. $V_{\text {lead }}$ is the output error signal when $t_{d}<t_{i}$ and $V_{l a g}$ is the output error signal when $t_{d}>t_{i}$.

computes whether $t_{i}<t_{d}$, while the circuit on the left computes whether $t_{i}>t_{d}$.

Let's first look at the circuit that creates $V_{\text {lag. }}$. The arrival of pulse1 triggers the delay element (as shown in Figure 1), and at the same time charges up node A. Node $\mathrm{B}$ does not go low until pulse 2 arrives. If dpulse1 arrives before pulse2, node $\mathrm{A}$ is discharged to ground and prevents node B from switching. Hence $V_{\text {lag }}$ stays low, whereas in the circuit on the left, $\overline{V_{\text {lead }}}$ becomes active. However if pulse 2 comes first before $d p u l s e 1$, node B goes low and $V_{\text {lag }}$ goes high. $V_{\text {lag }}$ is only high for the duration $\Delta t$. Q1 and Q2 are present to prevent $V_{\text {lag }}$ and $\overline{V_{\text {lead }}}$ from being active at the same time.

The circuit on the left creates $\overline{V_{\text {lead }}}$. When $\overline{V_{\text {lead }}}$ becomes active, Q2 turns on and the current through $\mathrm{Q} 1$ and $\mathrm{Q} 2$ is mirrored in $\mathrm{Q} 3$. This current prevents node $\mathrm{C}$ from being pulled low when $\overline{p u l s e 1}$ comes along, hence preventing $V_{\text {lag }}$ from becoming active.

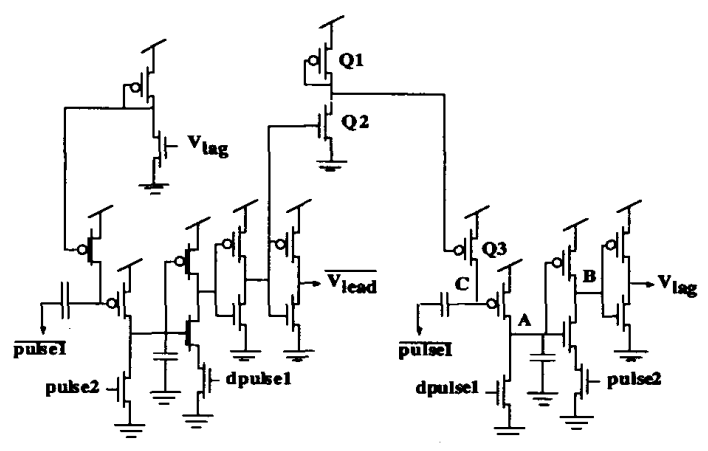

Figure 3: Lead/lag discriminator used in the adaptive delay system.

\section{Charge Pump}

The key block in the feedback system is the charge pump shown in Figure 4. The circuit adapts $V_{D}$ so that $t_{d}$ matches $t_{i}$. In traditional charge-pump phase-lock loop systems, a fixed current source is used to charge or discharge the capacitor $C_{b}$ during $\Delta t$. In our system however, the adaptation of $V_{D}$ depends on the delay contrast ratio, $\Delta t / t_{i}$. This ratio is important since for a given $\Delta t$ error, the amount of correction of $V_{D}$ should depend on the absolute temporal disparity, $t_{i}$. If $\Delta t$ is a small fraction of $t_{i}$, then $V_{D}$ should be corrected by a small amount. Conversely, if $\Delta t$ is a large fraction of $t_{i}$, then $V_{D}$ should be corrected by a larger amount. The dependence of the adaptation of $V_{D}$ on the delay contrast ratio allows the system to match $t_{D}$ with $t_{i}$ monotonically over a wide range of $t_{i}$.

The circuit operates as follows: The current flowing in Q4 is equal to the current $I_{i n}$ in the delay element since Q4 is also biased by $V_{D}$. This current is mirrored through transistor, Q5 into Q11 and is mirrored through transistors Q6-Q7 into Q9. Q8 and Q12 act as switches in the circuit. If $\overline{V_{\text {lead }}}$ is active, $V_{D}$ increases during $\Delta t$. Conversely when $V_{\text {lag }}$ is active, $V_{D}$ decreases instead.

When $\overline{V_{\text {lead }}}$ is active, the current $I_{D}$ charging up the capacitor is determined by $V_{D} . I_{D}$ is also equal to the capacitive current through the capacitor $C_{b}$. In subthreshold operation, $I_{D}$ can be expressed as $\left(V_{D}\right.$ here is referenced to $V_{d d}$ ):

$$
I_{D}=I_{0} e^{-\frac{\kappa V_{D}}{U_{T}}}=C_{b} \frac{d V_{D}}{d t}
$$

where $I_{0}$ is the leakage current, $\kappa$ is $\frac{C_{o x}}{C_{d}+C_{o x}}, C_{o x}$ is the gate oxide capacitance and $C_{d}$ is the depletion layer capacitance[2].

Let $t_{d 0}$ be the initial delay through the delay element. 


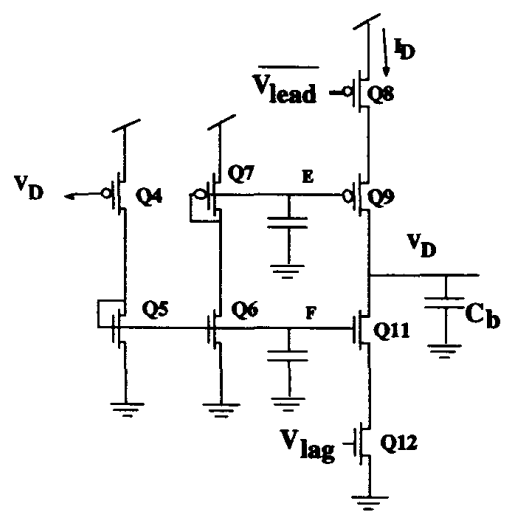

Figure 4: Charge pump used in the adaptive delay system. This circuit uses the error signals, $V_{\text {lead }}$ or $V_{\text {lag }}$, to correct the bias voltage, $V_{D}$. The update of $V_{D}$ depends on the duration of the error signals and on the current source controlled by $V_{D}$.

Integrating both sides of equation 1 , we get

$$
\begin{aligned}
& \int d t=\int \frac{C_{b} d V_{D}}{I_{D}} \\
\Delta t= & \int \frac{C_{b}}{I_{0} e^{-\frac{\kappa V_{D}}{U_{T}}}} d V_{D} \\
= & \frac{C_{b} U_{T}}{\kappa}\left[1 / I_{D 1}-1 / I_{D 0}\right]
\end{aligned}
$$

where $\Delta t$ is the duration of $\overline{V_{\text {lead }}}, I_{D 1}$ is the charging current in the delay element with bias voltage $V_{D 1}$ and $I_{D 0}$ is the charging current with bias voltage $V_{D 0}$. The bias voltage, $V_{D 1}$ creates a new delay through the delay element, $t_{d 1}$. We substitute $t_{d 1}$ and $t_{d 0}$ using the relationship derived in equation 6 in the variable delay element section into the above equation to get:

$$
\Delta t=\frac{C_{b} U_{T}}{\kappa C_{a} V_{d d}}\left[t_{d 1}-t_{d 0}\right]
$$

This equation allows us to express a relationship between the initial and final delays after the duration $\Delta t$.

$$
t_{d 1}=t_{d 0}+\Delta t \frac{\kappa C_{a} V_{d d}}{C_{b} U_{T}}=t_{d 0}+\gamma \Delta t
$$

where $\gamma=\frac{C_{a} V_{d d} \kappa}{C_{b} U_{T}}$. Ideally, we should set $\gamma=1$ for optimal response and no overshoot. The precision with which we can control $\gamma$ is limited by the repeatibility of $C_{a}, C_{b}, \kappa$ and $U_{T}$. Obviously if $\gamma>1$, the system is over-corrected and we would observe overshoots in the correction of the bias voltage.

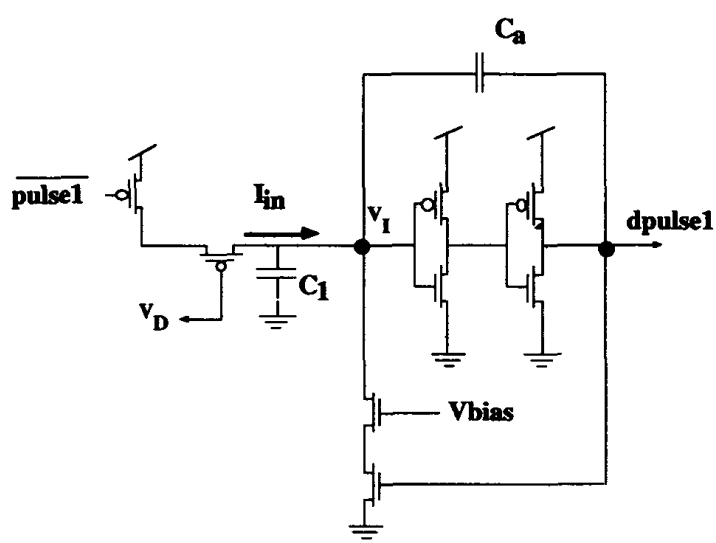

Figure 5: Variable delay element used in adaptive delay system. The delay is controlled by $V_{D}$.

\section{Variable Delay Element}

Initially, the output of the delay element, dpulse1, is at ground. Assume that $V_{I}$ must increase by $\Delta V_{I}$ before the delay element fires (i.e. dpulse1 goes to $V_{d d}$ ). The delay, $t_{d}$, through this element, which is the time before dpulse1 switches from ground to $V_{d d}$, can be described by:

$$
t_{d}=\frac{\Delta V_{I}}{d V_{D} / d t}
$$

The rate at which $V_{I}$ increases depend on the charging current, $I_{i n}$, i.e, $I_{i n}=C \frac{d V_{I}}{d t}$ where $C$ in this case is $C_{a}+C_{1}$. The final value of $\Delta V_{I}$ before the delay element fires is $\left(C_{a} V_{d d}\right) /\left(C_{a}+C_{1}\right)$. Substituting this expression back into equation 5 , we get:

$$
t_{d}=\frac{\frac{C_{a} V_{d d}}{C}}{\frac{I_{\text {in }}}{C}}=\frac{C_{a} V_{d d}}{I_{\text {in }}}
$$

\section{Experimental Results}

When the system is operating at steady state, $t_{d 0}$ is equal to the input temporal disparity, $t_{i 0}$. If the input temporal disparity increases from $t_{i 0}$ to $t_{i f}$, the change in input temporal disparity, $\Delta t_{0}$, is equal to $t_{i f}-t_{i 0}=t_{i f}-t_{d 0}$. The error signal on the $n$th iteration, $\Delta t_{n}$, tells us the error between the new input temporal disparity and the updated delay, $t_{i f}-t_{d n} . \Delta t_{n}$ is a geometric decay of $\Delta t_{0}$ as shown below:

$$
\Delta t_{n}=(1-\gamma)^{n} \Delta t_{0}
$$

$t_{d n}$ on the $n$th iteration is given by:

$$
t_{d n}=t_{d 0}(1-\gamma)^{n}+t_{i f}\left(1-(1-\gamma)^{n}\right)
$$




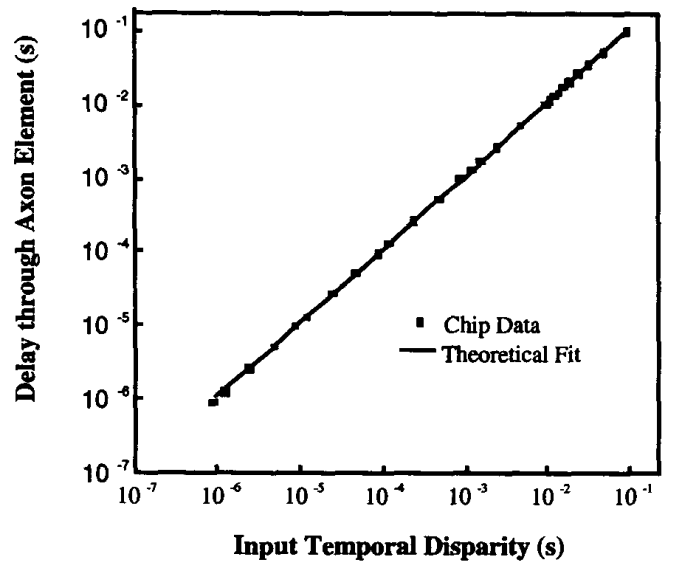

Figure 6: Measured delay through delay element as a function of input temporal disparity for system shown in Figure 1. The theoretical fit has a slope of one. The data shows a match over 5 decades.

From equation 7 , we observe that the error decreases geometrically as $n$ increases, i.e., $\lim _{n \rightarrow \infty} \Delta t_{n}=$ $0, \lim _{n \rightarrow \infty} t_{d n}=t_{i f}$. From equation 8 , we see that $t_{d n}$ approaches $t_{i f}$ as the number of iterations increases. We can get the updated bias voltage, $V_{D n}$, on the $n$th iteration by substituting the expression from equation 2 into equation 8 ,

$$
\begin{aligned}
V_{D n}= & V_{D 0}+ \\
& U_{T} \frac{\ln \left(\beta^{n}+e^{\kappa\left(V_{D f}-V_{D 0}\right) / U_{T}}\left(1-\beta^{n}\right)\right)}{\kappa}
\end{aligned}
$$

where $V_{D f}$ is the bias voltage corresponding to the delay, $t_{i f}$ and $\beta=(1-\gamma)$.

In the first experiment, we supplied two input signals, pulse 1 and pulse 2 with varying input temporal disparities to the system. For each $t_{i}$, the signals were repeated for several iterations. The interval, $t_{d}$, was measured when the system reaches steady-state. Figure 6 shows the $\log -\log$ plot of $t_{d}$ versus $t_{i}$. The points on the graph are the experimental data while the solid line is $t_{d}=t_{i}$. The adaptation happens over a range of 5 decades from $1 \mu s$ to $0.1 s$.

Next, we look at how $V_{D}$ changes over time when a sudden change in $t_{i}$ occurs. In this experiment, we provide 2 input clocks with a temporal disparity of $20 \mu s$ between pulse 1 and pulse 2 and decreased $t_{i}$ by $10 \%$. Since the input signals are presented over and over again, we can monitor the temporal change of $V_{D}$. Figure 7 shows the monotonic increase in $\Delta V_{D}$ as $V_{D}$ approaches its steadystate value. This experimental data shows that this system is stable. The smooth line in Figure 7 shows the theoretical fit through the experimental data. From this fit, $\gamma$ is about 0.03 which correlates well with the value

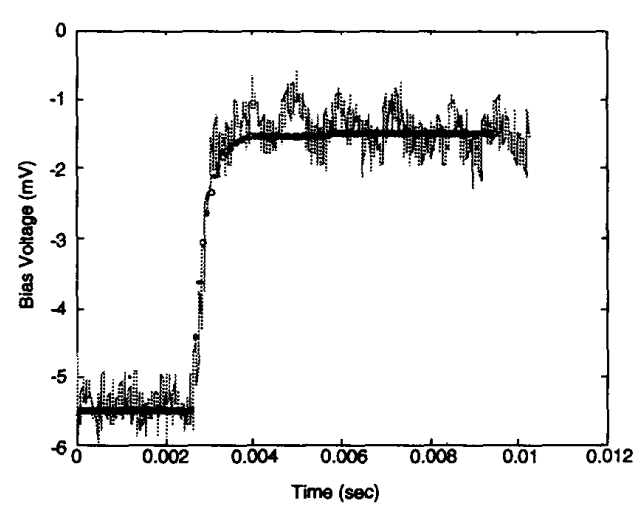

Figure 7: Change in bias voltage over time for a $10 \%$. change in delay contrast ratio.

computed from $\gamma=\frac{C_{a} V_{d d} \kappa}{C_{b} U_{T}}$.

\section{Conclusion}

We have developed an adaptive system that adjusts its delay through continuous-time adaptation to the temporal disparity between two input signals. The bias voltage to the delay element adapts monotonically towards the final steady-state value corresponding to the new input temporal disparity. It does not show the overshoots exhibited by conventional phase-locked loop systems. The system changes its adaptation dynamics depending on the delay contrast ratio in such a way that they are independent of the input temporal disparity itself. The new adaptation dynamics allow the system to have a lock-in range of over 5 decades in delay.

\section{Acknowledgements}

I would like to acknowledge Kwabena Boahen for multiple discussions during the development of this design; Tobi Delbrück, Dick Lyon and Rahul Sarpeshkar for insights and editorial inputs. I would also like to acknowledge the MOSIS foundation for the fabrication of this circuit.

\section{References}

[1] Gardner, Floyd M., "Charge-Pump Phase-Lock Loops", IEEE Transactions on Communications, COM-32, pp.1849-1858, 1980.

[2] Mead, C., Analog VLSI and Neural Systems, Addison-Wesley, 1989. 